\title{
Predictors of musculoskeletal pain in the upper extremities of individuals with spinal cord injury
}

\author{
DC Barbetta ${ }^{1}$, ACG Lopes ${ }^{1}$, FNMR Chagas ${ }^{1}$, PT Soares ${ }^{1}$, FM Casaro ${ }^{1}$, MF Poletto ${ }^{2}$, \\ YH de Carvalho Paiva Ribeiro ${ }^{1}$ and TO Ogashawara ${ }^{1}$
}

Introduction: Increased demands on the upper extremities (UE) have been associated with a higher occurrence of musculoskeletal pain in individuals with spinal cord injury (SCI).

Study design: This is a cross-sectional retrospective study.

Objective: The aim of this study was to characterize $\mathrm{SCl}$ subjects with musculoskeletal pain in the UE and to determine which variables could predict musculoskeletal pain in these individuals.

Setting: The study was conducted in SARAH Network of Rehabilitation Hospitals, Brasília, Brazil.

Methods: Five hundred sixty-four electronic medical records were investigated through a retrospective, exploratory and descriptive analysis to identify individuals with UE pain. A logistic regression model was applied to estimate (odds ratios) whether gender, age, the level of injury, severity of injury, time of injury, body mass index, type of mobility and locomotion aid could predict the occurrence of musculoskeletal pain.

Results: The prevalence of musculoskeletal pain in the UE was $27.7 \%$. The odds of having musculoskeletal pain were two times higher among woman and tetraplegic patients; those over 41 years of age had twice the frequency of pain than did those $<24.7$ years; $<1$ year of injury was a predictor of musculoskeletal pain, compared with the other quartiles (1.1-2.8, 2.9-6.8 and 6.9+ years of injury). There were no differences between the wheelchair and ambulatory individuals.

Conclusion: Female individuals, those with tetraplegia, aged $>40$ years and $<1$ year of injury showed an increased risk of musculoskeletal pain.

Spinal Cord (2016) 54, 145-149; doi:10.1038/sc.2015.126; published online 28 July 2015

\section{INTRODUCTION}

Individuals with spinal cord injury (SCI) have shown a better quality of life and, consequently, greater survival than they did a few decades ago. The global prevalence of SCI is estimated to be $\sim 236-1009$ cases per one million individuals per year. The estimated incidence in North America is 39 cases per million per year. ${ }^{1}$

As a consequence of lower extremity weakness or paralysis after an SCI, the upper extremities (UE) are used for daily living activities and to aid in locomotion. The increased use of the UE has been associated with a higher occurrence of musculoskeletal pain in this population, with a prevalence ranging from 5 to $78 \%$, based on cross-sectional surveys and out-patient evaluations. ${ }^{2-4}$

Dalyan et al., ${ }^{5}$ used a questionnaire to assess the interference of UE pain in the activities of daily living. Of the 130 subjects analyzed, $58.5 \%$ reported pain and $28 \%$ reported decreased independence. The pain interfered with activities such as transfer skills, pressure relief and wheelchair mobility, which require UE loading and/or repetitive movements. ${ }^{4-8}$

An epidemiological, etiological and pathomechanical literature review showed that complaints of pain in the shoulder are more common in tetraplegics, patients with complete injuries, females and individuals with advanced age. Some risk factors are include the time of injury, higher body mass index (BMI) and the use of a manual wheelchair. ${ }^{3}$

Subbarao et al., ${ }^{7}$ however, in a logistic regression analysis with a sample of 451 SCI veterans demonstrated that age, the neurological level and time of injury showed no significant difference in patients with or without pain.

Knowing the factors that contribute to the onset of musculoskeletal pain in the UE is important for establishing resources to prevent this condition in SCI patients.

Controversial results have been presented in the literature, which might be due to the influence of socio-cultural factors in the dependence/independence of patients.

The hypothesis of this study is that advanced age, length of time since injury and increased BMI have a significant role in upper limb musculoskeletal pain in manual wheelchair-using individuals with SCI because these factors are directly related to changes in the mechanical efficiency of UE joints and the amount of physical strain experienced during activities of daily living. Repetitive trauma that occurs in wheelchair transfers and outdoor wheeling appears to be the most common source of these conditions. ${ }^{3,9}$ Paraplegic individuals, who are more independent than tetraplegic patients, have an increased UE demand in daily activities and resultant overuse. Accordingly, men

${ }^{1}$ Spinal Cord Injury Neurorehabilitation Unit, SARAH Network of Rehabilitation Hospitals, Brasilia, Brazil and ${ }^{2}$ Spinal Cord Injury Neurorehabilitation Unit, SARAH Network of Rehabilitation Hospitals, Belo Horizonte, Brazil

Correspondence: DC Barbetta, Spinal Cord Injury rehabilitation SARAH Network of Rehabilitation Hospitals, SMHS Quadra 301 Bloco A, Brasília 70.335-901, DF, Brazil.

E-mail: dcbarbetta@sarah.br

Received 23 January 2015; revised 17 June 2015; accepted 23 June 2015; published online 28 July 2015 
with SCI have a greater risk of UE pain than do women. The lower activity level in tetraplegic patients and women might contribute to these subjects having no pain or a low level of pain. Regarding the completeness of injury, our hypothesis is that subjects with a complete motor injury have a greater chance of UE pain than do those with an incomplete motor injury because they have greater motor function preserved below the neurological level, ${ }^{10}$ which might include lower limb function and might decrease the amount of strength in and overuse of the UE. In addition, individuals with a complete motor injury are more wheelchair dependent. The biomechanical requirements for wheelchair propulsion and transfers might increase the shoulder and wrist effects. ${ }^{3}$

\section{Main objective}

The aim of this study was to determine the predictors of UE musculoskeletal pain in spinal cord injury patients.

\section{Secondary objective}

To characterize the SCI patients with a report of musculoskeletal pain in the upper extremities as well as the main joints involved.

\section{METHODS}

\section{Study design}

This is a cross-sectional, retrospective study.

\section{Participants}

We investigated the medical records, from The Quality Control Department of the SARAH Network of Rehabilitation Hospitals in Brasília, of 574 SCI inpatients and out-patients, who were followed after visiting the hospital between January and July 2010 . The inclusion criteria were traumatic-SCI patients with UE musculoskeletal pain without other comorbidities, such as neuropathic pain, peripheral nerve injury or upper extremity fractures. Patients with BrownSequard syndrome, central cord syndrome or grade E on the American SCI Association Impairment Scale (AIS) ${ }^{10}$ were excluded. The conus medullaris/ cauda equina syndrome was included.

\section{Study variables}

The following variables were identified: gender; age; the level of injury; completeness of injury; time of injury; BMI (weight/(heigh $\times$ height)); locomotion and mobility aid; musculoskeletal pain report and location of pain; the data were collected retrospectively from the medical records.

Upper extremity musculoskeletal pain was considered the variable of interest and was treated as a simple dichotomous variable (yes or no). This variable was investigated in the data from the physiotherapy and occupational therapy patient records. The medical records of all patients were investigated to retrieve the reports of musculoskeletal pain before January-July 2010. If the patient had more than one complaint of pain, the first record was considered.

The other variables (gender; age, the level of injury; completeness of injury; time of injury; BMI; locomotion and mobility aid) were considered independent variables and were collected when the pain was reported.

The level and completeness of injury were measured by a physician using the AIS. ${ }^{10}$ The neurological level was classified as the 'most caudal segment of the spinal cord with normal sensory and motor function', through the evaluation of dermatomes and myotomes. For the completeness of injury, the preservation of sensory and/or motor function below the neurological level were evaluated, including evaluation of the sacral segments (S4-5). ${ }^{10}$ The level of injury was divided into high tetraplegia (C1-5), low tetraplegia (C6-1), high paraplegia (T2-6) and low paraplegia (T7-S5). When a difference between the sides was observed, the most rostral level of the injury was considered; the higher the injury was on the spinal cord, the greater the functional impairment and, consequently, the more extensive the demands were on the upper extremity. The completeness of injury was assessed according to the AIS, as follows: A (a complete motor and sensory lesion), B (preservation of sensory function, without preservation of motor function), C and D (motor function preserved below the level of the lesion). ${ }^{10}$

Percentiles based on quartiles were used to classify the age and the time of injury. The age was divided into the following intervals: $<24.7$ years, $24.8-30.9$ years, $31.0-40.9$ years and $41+$ years. The time of injury was separated into the following quartiles: injury of $<1$ year, $1.1-2.8$ years, $2.9-6.8$ years and $6.9+$ years. BMI was treated as a continuous variable. The other variables were gender, the level and completeness of injury and the locomotion and mobility aid; these variables were treated as categorical variables.

In addition, the subjects were classified according to their use of locomotion and mobility aids. Regarding locomotion, the groups were divided into wheelchair users, household ambulators and community ambulators. We divided wheelchair users into the following three categories: (1) wheelchair use and absence of orthostatism; (2) wheelchair use and orthostatism; and (3) wheelchair use and walking exercises because of the increased demand to the UE involved in the process of transferring to the standing position and in the process of remaining in the position and walking. ${ }^{11}$ Regarding mobility aid, the patients were classified into the (1) wheelchair, (2) parallel bars, (3) walker, crutches (unilateral or bilateral), (4) cane (unilateral) and (5) those that did not use mobility aids groups.

\section{Statistical analysis}

Descriptive and exploratory data analyses were performed to demonstrate the relationship between pain and the independent variables. Student's $t$-test was applied for the continuous variables, and the equivalence among the groups was assessed using Levene's test. The associations between the 'pain report' and the categorical variables were tested using the $\chi^{2}$ test and, when necessary, Fisher's exact test.

A logistic regression model was applied to calculate the odds ratios. In cases in which the independent variables comprised more than two categories, a dummy variable was applied, using as the reference the category with the decreased perception of pain, in accordance with a preliminary exploratory analysis.

The explanatory variables that were statistically significant $(P$-value $<0.10)$ in the simple regression analysis were tested together in a multiple regression analysis using the backward stepwise selection method based on the likelihood ratio, with a $5 \%$ tolerance to introduce the variable in the model and a $10 \%$ tolerance to exclude it.

The Statistical Package for Social Sciences, version 13.0 (SPSS, Inc., Chicago, IL, USA), was used for the analysis.

\section{RESULTS}

Of the 574 patient records studied, 10 patients with Brown-Sequard syndrome, central spinal cord syndrome or AIS E were excluded. Of the 564 patients who participated in the study, $79 \%$ were male, with an average age of 34 years (range 14-79 years), a mean BMI of $22.4 \pm 4.8 \mathrm{~kg} \mathrm{~m}^{-2}$ and an average of 5 years of injury on average (range $0.4-46$ years); $61 \%$ of the participants had paraplegia, and $71.3 \%$ had a complete motor injury (AIS A and B).

The prevalence of musculoskeletal UE pain was $27.7 \%$ (156 patients), with a confidence interval of $24.0-31.4 \%$ (confidence level: 0.95). In total, 256 joints were affected, with a $69,19,9$ and $3 \%$ prevalence of shoulder, wrist, elbow and hand pain, respectively. In the pain group, $29 \%$ of the patients were female, with a mean age of $36 \pm 13.4$ years and $4.8 \pm 7.1$ years of injury, and the individuals presented predominantly with low tetraplegia, a complete motor lesion and use of a wheelchair as the primary mobility aid (Table 1).

In a comparison of the groups with and without pain, age, the BMI and the mobility aid showed no statistically significant difference. The subgroups of the patients using crutches and canes as well as those that did not use any mobility aid were pooled for the comparative analysis; however, no difference was observed in these patients with or without pain. The other variables, including gender, the time of injury, the 
level and completeness of injury, and locomotion, were significantly different and were submitted to a multiple regression analysis. Although age did not show a significant difference in the comparison within the groups, it was considered clinically relevant and included in the regression analysis (Table 2). The multiple regression analysis showed that women are twice as likely to experience UE pain as men. Individuals aged 41 or more years are twice as likely to have pain as are patients $<24.7$ years of age. Regarding the time of injury, individuals with injury that has lasted $<1$ year have a greater risk of pain than do the individuals in other quartiles; the former are two times more likely to experience UE pain than are individuals with 1.1-2.8 years of injury and three times more likely than individuals with 2.9-6.8 and 6.9+ years of injury. The tetraplegic patients had almost twice the risk of pain than did the paraplegics. The individuals exclusively using

Table 1 Comparative analysis of the groups with and without pain

\begin{tabular}{|c|c|c|c|}
\hline $\begin{array}{l}\text { Characteristics of the sample } \\
(n=564)\end{array}$ & With pain & Without pain & P-value \\
\hline Female gender, $n(\%)$ & $45(29)$ & $75(18)$ & 0.007 \\
\hline Age (years), mean (s.d.) & $36.0(13.4)$ & $32.9(11.4)$ & \\
\hline median (Q1-Q3) & $32.5(26.2-44.3)$ & $30.2(24.2-38.8)$ & \\
\hline Age (years), n (\%) & & & 0.185 \\
\hline$\leqslant 24.7$ & $31(22)$ & $110(79)$ & \\
\hline $24.8-30.9$ & $37(26)$ & $104(74)$ & \\
\hline $31.0-40.9$ & $41(29)$ & $100(71)$ & \\
\hline $41.0+$ & $47(33)$ & $94(67)$ & \\
\hline Time of injury (years), mean (s.d.) & $4.8(7.1)$ & $5.4(6.2)$ & \\
\hline median (Q1-Q3) & $1.7(0.5-5.9)$ & $3.2(1.3-7)$ & \\
\hline Time of injury (years), n (\%) & & & $<0.001$ \\
\hline$\leqslant 1.0$ & $61(43)$ & $80(57)$ & \\
\hline $1.1-2.8$ & $33(23)$ & $108(77)$ & \\
\hline $2.9-6.8$ & $28(20)$ & $113(80)$ & \\
\hline $6.9+$ & $34(24)$ & $107(76)$ & \\
\hline BMI, mean (s.d.) & $22.5(5.3)$ & $21.9(4.7)$ & 0.312 \\
\hline Level of injury, $n(\%)$ & & & $<0.001$ \\
\hline C1-5 & $9(6)$ & $23(6)$ & \\
\hline C6-1 & $76(49)$ & $113(28)$ & \\
\hline T2-6 & 30 (19) & $116(28)$ & \\
\hline T7-S5 & $41(26)$ & $156(38)$ & \\
\hline AIS, $n(\%)$ & & & 0.039 \\
\hline$A$ & $81(52)$ & $236(58)$ & \\
\hline B & $31(20)$ & $54(13)$ & \\
\hline C & $19(12)$ & $29(7)$ & \\
\hline $\mathrm{D}$ & $14(9)$ & $40(10)$ & \\
\hline Conus medullaris/cauda equina & $11(7)$ & $49(12)$ & \\
\hline Locomotion, $n(\%)$ & & & $<0.001$ \\
\hline Wheelchair users & $99(64)$ & $168(41)$ & \\
\hline Wheelchair+orthostatism & $23(15)$ & $105(26)$ & \\
\hline Wheelchair+walking exercise & $17(11)$ & 76 (19) & \\
\hline Household ambulators & $8(5)$ & $23(6)$ & \\
\hline Community ambulators & $9(6)$ & $36(9)$ & \\
\hline Mobility aid, n (\%) & & & 0.101 \\
\hline Wheelchair & $116(74)$ & $261(64)$ & \\
\hline Parallel bars & $10(6)$ & $44(11)$ & \\
\hline Walkers & $19(12)$ & $60(15)$ & \\
\hline Crutches & $4(3)$ & $24(6)$ & \\
\hline Canes & $3(2)$ & $14(3)$ & \\
\hline No mobility aid & $4(3)$ & $5(1)$ & \\
\hline
\end{tabular}

AIS: American Spinal Cord Injury Association Impairment Scale; BMI: body mass index; C: cervical; L: lumbar; n: numberQ1: Quartile 1; Q3: Quartile 3; T: thoracic wheelchairs for locomotion had twice the risk of pain than did those in the 'wheelchair+orthostatism' and 'wheelchair+walking exercise' groups. There was no difference in a comparison with the ambulators. The household and community ambulators were pooled for the comparative analysis, and no differences were observed in the results.

\section{DISCUSSION}

This study showed characteristics that might be associated with musculoskeletal UE pain in individuals with SCI, such as female gender, age over 41 years, $<1$ year of injury, low tetraplegia, a complete motor injury and wheelchair users.

Caution is needed in comparing this study with the results presented in the literature because our population tended to be younger (34 years on average) and to have a shorter time of injury (5 years on average). The heterogeneity of the results could be attributed to the methodologies adopted in the studies. Many studies do not discriminate between musculoskeletal pain and neuropathic pain.

The joint most affected was the shoulder, which is consistent with the findings in the literature, with a prevalence of up to $70 \%{ }^{3,5,6,12}$ After SCI, the UE demonstrate failure in primary grip activities and begin to experience an overload in the performance of repetitive movements, such as transferring, pushing up and propelling the wheelchair. ${ }^{6,11}$ There were some differences regarding the most affected joints between paraplegics and tetraplegics, including wrist and shoulder pain, respectively, as the most common complaint. ${ }^{13}$

Age has been positively associated with ongoing shoulder pain that is increased in patients aged 31-40 years, with an increased risk of 5\% per year. ${ }^{5,8}$ The viscoelastic properties of the shoulder ligaments and capsule might change with age and might impair the performance of daily living activities and mobility efficiency. ${ }^{3}$

In this study, the individuals with injury lasting $<1$ year had a greater risk of UE pain. Between the first 3 months of in-patient rehabilitation and discharge, UE musculoskeletal pain showed a decrease of $30 \% .{ }^{14}$ Seventy-five percent of the tetraplegic patients reported shoulder pain during the initial rehabilitation, and $78 \%$ of them were pain free at discharge. ${ }^{15}$

A prospective cohort study showed that $70 \%$ of the subjects presented a 'Decreased or No/Low pain' trajectory compared with $30 \%$ with a 'High pain' trajectory, as measured 5 years after discharge. ${ }^{16}$

Some authors have stated that the adaptation process of gaining new UE skills after an SCI could lead to musculoskeletal pain. Subjects with a longer time of injury gain ability in and muscle strength for performing daily living activities and thus experience reduced pain. ${ }^{14}$ The reduction in reported pain might be influenced by a decrease in the activity level because of aging. ${ }^{13}$

Regarding the level of injury, this study showed a two-fold greater risk of UE pain in tetraplegics, which corroborates the findings presented in the literature. This risk appears to be associated with the following factors: immobilization or a reduction in the range of motion in the acute phase, which leads to muscle shortening and shoulder capsule tightness; impaired muscle strength and balance resulting from weakness; paralysis resulting from partial innervations; and spasticity, which could lead to variations in the pathogenesis of pain among tetraplegic and paraplegic individuals. ${ }^{3,14,14-17}$

The completeness of the injury was an additional predictor of UE pain. Some authors have associated shoulder pain with the overuse/ overload experienced in wheelchair propulsion and transfers, and with the decreased postural trunk control that is related to the decreased 
Table 2 Logistic regression for association between pain and the characteristics of the individuals

\begin{tabular}{|c|c|c|c|}
\hline Variables & Odds ratio* & \multicolumn{2}{|c|}{ Cl 95\% } \\
\hline \multicolumn{4}{|l|}{ Gender } \\
\hline Male & 1.0 & & \\
\hline Female & 2.2 & 1.3 & 3.4 \\
\hline \multicolumn{4}{|l|}{ Age (years) } \\
\hline$\leqslant 24.7$ & 0.5 & 0.3 & 0.9 \\
\hline 24.8-30.9 & 0.8 & 0.4 & 1.4 \\
\hline 31.0-40.9 & 0.8 & 0.5 & 1.5 \\
\hline $41.0+$ & 1.0 & & \\
\hline \multicolumn{4}{|l|}{ Time of injury (years) } \\
\hline$\leq 1$ & 1.0 & & \\
\hline $1.1-2.8$ & 0.4 & 0.2 & 0.7 \\
\hline $2.9-6.8$ & 0.3 & 0.2 & 0.5 \\
\hline $6.9+$ & 0.3 & 0.2 & 0.6 \\
\hline \multicolumn{4}{|l|}{ Level of injury } \\
\hline Paraplegia & 1.0 & & \\
\hline Tetraplegia & 1.9 & 1.2 & 3.0 \\
\hline \multicolumn{4}{|l|}{ Locomotion, n (\%) } \\
\hline Wheelchair users & 1.0 & & \\
\hline Wheelchair + orthostatism & 0.5 & 0.3 & 0.8 \\
\hline Wheelchair + walking exercise & 0.5 & 0.3 & 1.0 \\
\hline Household ambulators & 0.6 & 0.3 & 1.5 \\
\hline Community ambulators & 0.5 & 0.2 & 1.1 \\
\hline
\end{tabular}

Abbreviations: $\mathrm{Cl}$, confidence interval $\mathrm{n}$, number.

*Logistic regression model.

preservation of the motor unit. ${ }^{3}$ The 'High pain' trajectory over 5 years applied to more patients with lower muscle strength than did the 'No or Low pain' trajectory. ${ }^{16}$

Our study showed that women are twice as likely to experience pain in the UE, which was in agreement with a literature review by Dyson et al. ${ }^{3}$ Women have physiological and anatomical differences in the shoulder and apply a higher percentage of force to propel a wheelchair than do men, which increases the risk for rotator cuff abnormalities, edema, arthrosis, subacromial spur formation and coracoacromial ligament thickening. ${ }^{3,18}$ A study that analyzed the difference in the aging process of men and women with SCI, controlling for confounders such as age (older than 40 years) and time of injury (at least 20 years), found no difference in the relationship to chronic pain complaints between the genders. However, in the qualitative analysis, women reported that pain affected the performance of activities of daily living and presented significantly increased hand pain, which was associated with arthritis. ${ }^{19}$

The influence on the reported pain of assuming an orthostatic position and performing walking exercises was investigated; these activities involve strength and repetitive UE movements that impose increased biomechanical demands on the upper limb joints and muscles. Pain reports were more frequent in wheelchair-dependent patients who do not stand or perform gait exercises. Bias should be considered with regard to the restrictions imposed by pain or overload during the standing and walking exercises in our program.

The literature supports a $67 \%$ incidence of shoulder pain that starts from the initial phases of wheelchair dependency. ${ }^{8}$ The biomechanics of wheelchair propulsion, which include extreme positions of the shoulder such as extreme internal rotation and abduction, might predispose to shoulder impingement, particularly with a narrowed humeroacromial space. ${ }^{2,20}$ Otherwise, the orthostatic posture allows the shoulder joint to remain in the most favorable position, with a less anterior tilt of the scapula and internal rotation of the glenohumeral joint. ${ }^{11}$ The use of a mobility aid (wheelchair, parallel bars, walkers, crutches and canes) was not considered a predictive factor of shoulder pain.

The UE biomechanics of hand rim wheelchair propulsion combined with the skeletal muscle overload demands during transfer activities, require more effort from a heavier person. ${ }^{3,14}$ Despite the trend of a higher BMI being an important predictor of musculoskeletal UE pain, ${ }^{14,16}$ it was not considered statically significant in this survey. This finding is consistent with the results of McCasland et al. ${ }^{12} \mathrm{~A}$ regression analysis was used to evaluate the relationship between the injury level and completeness to the occurrence of UE pain. Age, gender and the BMI were considered confounding variables. Older individuals, with incomplete lesions, a higher FIM motor score and a higher BMI had an increased risk of UE pain. ${ }^{14}$ Our population had, on average, a normal BMI $\left(22.4 \pm 4.8 \mathrm{~kg} \mathrm{~m}^{-2}\right)$ and $\sim 68 \%$ of the inpatients had a BMI $<25 \mathrm{~kg} \mathrm{~m}^{-2}$, which could influence the results.

This retrospective and cross-sectional design study has some limitations. A retrospective analysis could lead to informational retrieval bias resulting from errors in measurement or data acquisition. Observational analysis does not allow us to establish a causal nexus. In addition, other important factors that were not addressed in this study might influence the onset of musculoskeletal pain in individuals with SCI, including the following: the level of functional independence; the features of the wheelchair used (including the weight and the positioning of the rear axle); spasticity; and other factors with equal or greater importance.

\section{CONCLUSION}

Musculoskeletal pain in the upper extremities of SCI patients is an incapacitating condition. Characteristics related to the individual and to the SCI are key factors, which should be established. Despite the difficulty of comparing studies, variables such as age, gender, the level of injury, the time of injury and the use of a wheelchair should be highlighted in physical evaluations to anticipate possible complaints of pain both in the rehabilitation setting and in everyday life. These factors demonstrate a clear influence on the pain process and should be considered in future studies. The research process is essential to understanding the biomechanics involved in task performance and to optimizing the interventional resources, which could protect the upper limb joints and prevent pain.

Musculoskeletal pain and its etiology should not be minimized by considerations regarding the loss of motion and bladder and bowel changes, among other challenges for SCI patients. On the basis of this study, we initiated an awareness of complaints of pain by SCI patients as well as adequate physical and functional assessment processes for these patients.

This study, in addition to our critical literature review, ${ }^{2,3,8,12}$ is beneficial to our rehabilitation team for promoting assistance for in-patients and out-patients. Our current focus includes the following aims: preventing UE pain and increasing the knowledge related to UE biomechanics; implementing appropriate daily live activity techniques, including wheelchair propulsion, avoiding overuse and weight-bearing; designing a balanced muscle fitness program for UE; establishing a nutritional orientation aimed at weight control; developing a prescription of the wheelchair and defining postural optimization; and, 
evaluating the indication for adaptive devices and environmental changes.

\section{DATA ARCHIVING}

There were no data to deposit.

\section{CONFLICT OF INTEREST}

The authors declare no conflict of interest.

1 Cripps RA, Lee BB, Wing P, Weerts E, Mackay J, Brown D. A global map for traumatic spinal cord injury epidemiology: towards a living data repository for injury prevention. Spinal Cord 2011; 49: 493-501.

2 Boninger ML, Waters RL, Chase T, MPJM Dijkers, Gellman H, Gironda RJ et al. Preservation of upper limb function following spinal cord injury: a clinical practice guideline for health-care professionals. J Spinal Cord Med 2005; 28: 433-470.

3 Dyson-Hudson TA, Kirshblum SC. Shoulder pain in chronic spinal cord injury, Part I: epidemiology, etiology, and pathomechanics. J Spinal Cord Med 2004; 27: 4-17.

4 Capoor J, Stein AB. Aging with spinal cord injury. Phys Med Rehabil Clin N Am 2005 , 16: 129-161.

5 Dalyan M, Cardenas DD, Gerard B. Upper extremity pain after spinal cord injury. Spinal Cord 1999; 37: 191-195.

6 Gellman H, Sie I, Waters RL. Late complications of the weight-bearing upper extremity in the paraplegic patient. Clin Orthop Relat Res 1988; 233: 132-135.

7 Subbarao JV, Klopfstein J, Turpin R. Prevalence and impact of wrist and shoulder pain in patients with spinal cord injury. J Spinal Cord Med 1995; 18: 9-13.

8 Alm M, Saraste H, Norrbrink C. Shoulder pain in persons with thoracic spinal cord injury: prevalence and characteristics. J Rehabil Med 2008; 40: 277-283.
9 Pentland WE, Twomey LT. Upper limb function in persons with long term paraplegia and implications for independence: Part I. Paraplegia 1994; 32 211-218.

10 American Spinal Injury Association. International standards for neurological and functional classification of spinal cord injury, Revised 2000Chicago: ASIA2000.

11 Riek LM, Ludewig PM, Nawoczenski DA. Comparative shoulder kinematics during free standing, standing depression lifts and daily functional activities in persons with paraplegia: considerations for shoulder health. Spinal Cord 2008; 46: 335-343.

12 McCasland LD, Budiman-Mak E, Weaver FM, Adams E, Miskevics S. Shoulder pain in the traumatically injured spinal cord patient: evaluation of risk factors and function. $J$ Clin Rheumatol 2006; 12: 179-186.

13 Sie IH, Waters RL, Adkins RH, Gellman H. Upper extremity pain in the postrehabilitation spinal cord injured patient. Arch Phys Med Rehabil 1992; 73: 44-48.

14 van Drongelen S, de Groot S, Veeger HE, Angenot EL, Dallmeijer AJ, Post MW et al. Upper extremity musculoskeletal pain during and after rehabilitation in wheelchair-using persons with a spinal cord injury. Spinal Cord 2006; 44 152-159.

15 Waring WP, Maynard FM. Shoulder pain in acute traumatic quadriplegia. Paraplegia 1991; 29: 37-42.

16 Eriks-Hoogland IE, Hoekstra T, de GS, Stucki G, Post MW, van der Woude LH. Trajectories of musculoskeletal shoulder pain after spinal cord injury: Identification and predictors. J Spinal Cord Med 2014; 37: 288-298.

17 Curtis KA, Drysdale GA, Lanza RD, Kolber M, Vitolo RS, West R. Shoulder pain in wheelchair users with tetraplegia and paraplegia. Arch Phys Med Rehabil 1999; 80: 453-457.

18 Boninger ML, Dicianno BE, Cooper RA, Towers JD, Koontz AM, Souza AL. Shoulder magnetic resonance imaging abnormalities, wheelchair propulsion, and gender. Arch Phys Med Rehabil 2003; 84: 1615-1620.

19 McColl MA, Charlifue S, Glass C, Lawson N, Savic G. Aging gender, and spinal cord injury. Arch Phys Med Rehabil 2004; 85: 363-367.

20 Ambrosio F, Boninger ML, Souza AL, Fitzgerald SG, Koontz AM, Cooper RA. Biomechanics and strength of manual wheelchair users. J Spinal Cord Med 2005; 28 407-414. 